\title{
Confiabilidade da avaliação da visão funcional para crianças com baixa visão de dois a seis anos - em busca de evidências
}

\author{
Reliability of functional vision assessment in children with low vision from \\ 2 to 6 years old - searching for evidences
}

Luciana Drummond de Figueiredo Rossi ${ }^{1}$, Galton Carvalho de Vasconcelos ${ }^{2}$, Grace Rego Saliba ${ }^{3}$, Lívia de Castro Magalhães ${ }^{4}$, Angela Maria Anselmo Soares ${ }^{5}$, Silvia Santiago Cordeiro ${ }^{6}$, Regina Helena Caldas de Amorim ${ }^{4}$

\section{RESUMO}

Objetivos: Avaliar a confiabilidade interexaminadores, fazer o teste-reteste e verificar a consistência interna da avaliação da visão funcional para crianças com baixa visão de dois a seis anos de idade (AVIF-2 a 6 anos).

Métodos: Foram testados sete domínios: fixação visual, seguimento visual, campo visual de confrontação, coordenação olho-mão, visão de contraste, deslocamento no ambiente e visão de cores, em 40 crianças, 20 com baixa visão e 20 sem alterações visuais. A consistência interna foi examinada para o teste de todas as crianças e a confiabilidade interexaminadores e teste-reteste para 12 delas.

Resultados: Seis domínios apresentaram ao teste-reteste coeficiente de correlação intraclasse com valores de 0,5361 a 1,000. Para o domínio deslocamento no ambiente esse coeficiente foi de 0,37. Na confiabilidade interexaminadores, somente o campo visual de confrontação apresentou valor abaixo do esperado $(0,3901)$. O coeficiente kappa ponderado obteve resultados que variaram de $-0,087$ a 1,000. O valor do alfa de Cronbach variou de 0,584 (no deslocamento no ambiente) a 0,973 (na visão de contraste)

Conclusão: A AVIF-2 a 6 anos apresentou bons índices de confiabilidade, mas a confiabilidade dos domínios, quando analisados isoladamente, precisa ser aprimorada.

Descritores: Baixa visão; Transtornos da visão; Testes visuais; Acuidade visual; Visão ocular/fisiologia; Desenvolvimento infantil; Estudos de validação; Pré-escolar

\begin{abstract}
Purpose: The functional vision assessment (AVIF-2 to 6 years) for low vision children with ages from 2 to 6 years old was analyzed. The inter-rater, test-retest and the internal consistency were verified for reliability.

Methods: The test, composed by seven domains (visual fixation, visual following, visual field confrontation, eye-hand coordination and surrounding locomotion, contrast vision and color vision discrimination), was applied to 40 children, 20 with low vision and 20 without low vision. The inter-rater and test-retest reliability were analyzed with 12 children and the internal consistency with 40 children.
\end{abstract}

Results: Concerning test-retest reliability, six domains exhibited intra-class correlation coefficients ranging from 0.5361 to 1.000 . For the movement within surroundings category, this coefficient was 0.37 . For inter-rater reliability, only the category of confrontation visual field was bellow expectations (0.3901). The weighted kappa coefficient exhibited values ranged from -0.087 to 1.000. Cronbach's alfa ranged from 0.584 (movement within surroundings) to 0.973 (contrast sensitivity).

Conclusion: AVIF-2 to 6 years presented good reliability indexes, however a few items within the domains need minor adjustments in order to improve scores' consistency of some items.

Keywords: Vision, low; Vision disorders; Vision tests; Visual acuity; Vision, ocular/physiology; Childhood development; Validation studies; Child, preschool

\section{INTRODUÇÃO}

Embora existam alguns protocolos para avaliação da visão funcional de crianças, a maioria não avançou em termos de validação, além de haver variações quanto à amostra e idade de aplicação(1-10) A avaliação da visão funcional (AVIF) 2 a 6 anos $^{(11)}$ foi criada para crianças de dois a seis anos de idade com baixa visão e é utilizada para avaliar e programar a intervenção visual precoce.

Para se avaliar a qualidade de um teste aplicado à visão, é necessário estudar-se a confiabilidade do instrumento, que pode ser definida como a precisão da medida dos escores ao longo do tempo e por diferentes examinadores. O coeficiente de confiabilidade é uma estimativa de quanto o resultado observado reflete o escore verdadeiro. O coeficiente de confiabilidade, que varia de 0 a 1,00, corresponde à proporção da variância do escore observado que é atribuída à variância do escore verdadeiro ${ }^{(12,13)}$.

Existem vários índices de confiabilidade: teste-reteste, interexaminadores e consistência interna dos itens. A confiabilidade testereteste, se refere à estabilidade temporal do instrumento e a confiabi-

Submetido para publicação: 25 de abril de 2012

Aceito para publicação: 26 de junho de 2012

Trabalho realizado no Setor de Baixa Visão Infantil, Hospital São Geraldo, Hospital das Clínicas, Universidade Federal de Minas Gerais - UFMG - Belo Horizonte (MG), Brasil.

Fisioterapeuta, Setor de Baixa Visão Infantil, Hospital São Geraldo, Hospital das Clínicas, Universidade Federal de Minas Gerais - UFMG - Belo Horizonte (MG), Brasil.

2 Médico, Coordenador do Setor de Baixa Visão Infantil, Hospital São Geraldo, Hospital das Clínicas, Universidade Federal de Minas Gerais - UFMG - Belo Horizonte (MG), Brasil.

3 Terapeuta Ocupacional, Setor de Baixa Visão Infantil, Hospital São Geraldo, Hospital das Clínicas, Universidade Federal de Minas Gerais - UFMG - Belo Horizonte (MG), Brasil.

${ }_{4}$ Professor, Universidade Federal de Minas Gerais - UFMG - Belo Horizonte (MG), Brasil.

Médico, Setor de Baixa Visão Infantil, Hospital São Geraldo, Hospital das Clínicas, Universidade

Federal de Minas Gerais - UFMG - Belo Horizonte - (MG), Brasil.

${ }^{6}$ Médico, Prefeitura Municipal de Belo Horizonte, Minas Gerais (MG), Brasil.

Financiamento: Não houve financiamento para este trabalho.

Divulgação de potenciais conflitos de interesse: L.D.F. Rossi, Nenhum; G.C.Vasconcelos, Nenhum; G.R.Saliba, Nenhum; L.C.Magalhães, Nenhum; A.M.A.Soares, Nenhum; S.S.Cordeiro, Nenhum; R.H.C.Amorim, Nenhum.

Endereço de correspondência: Luciana Drummond de Figueiredo Rossi, Alameda Adamello, 249, Condomínio Villa Alpina - Nova Lima (MG) - 34000-000 - Brasil - E-mail: lucianarossi@ig.com.br ETIC 684/07 - Universidade Federal de Minas Gerais - UFMG - Belo Horizonte (MG), Brasil. 
lidade interexaminadores é testada quando dois examinadores diferentes pontuam o teste de um mesmo indivíduo. A consistência interna corresponde ao grau de correlação entre os itens de uma escala(14).

Poucos foram os trabalhos sobre avaliação da visão funcional que incluíram análises de confiabilidade. Blanksby e Langford ${ }^{(2)}$, com relação ao VAP-CAP (Visual Assessment Procedure-Capacity, Attention and Processing), demonstraram que a confiabilidade teste-reteste apresentou valor médio de correlação de 0,97 para os escores totais. Na confiabilidade interexaminadores, a correlação média foi de 0,99.

A consistência interna dos itens foi verificada para o Children's Visual Function Questionnaire (CVFQ) ${ }^{(6)}$, um questionário de qualidade de vida relacionada à visão. Os autores relataram valores de alfa de Cronbach de 0,60 a 0,86 para os seis domínios do teste (saúde geral, saúde geral da visão, competência, personalidade, impacto familiar e tratamento). No domínio competência, que fornece dados sobre a visão funcional, o valor de alfa foi de 0,70 , na versão para crianças com menos de três anos, e 0,77 para crianças de três a sete anos.

Alguns autores em um método de avaliação neonatal, relataram boa confiabilidade interexaminadores, porém sem análises estatísticas. Ricci et al. mostraram que em 12 dos 13 itens da avaliação ocorreu concordância superior a 95\% entre cada observador e o examinador principal, e entre dois observadores individuais ${ }^{(9,10)}$.

A porcentagem de crianças incluídas na análise de confiabilidade nos estudos prévios é apresentada no quadro 1.

Dada a importância da utilização de um instrumento com propriedades psicométricas adequadas, o objetivo do presente estudo foi analisar a confiabilidade interexaminadores, teste-reteste e a consistência interna da AVIF-2 a 6 anos. Com base nessa análise, pode-se fazer os ajustes necessários, para disponibilizá-la para uso clínico.

\section{MÉTODOS}

A confiabilidade da AVIF-2 a 6 anos foi estudada em 20 crianças do Setor de Baixa Visão Infantil do Hospital São Geraldo - Hospital das Clínicas da UFMG e em 20 controles. Foi analisada a confiabilidade para o escore total do teste e para os sete domínios: fixação visual, seguimento visual, campo visual de confrontação, coordenação olho-mão, visão de contraste, deslocamento no ambiente e visão de cores.

A confiabilidade interexaminadores foi realizada com 12 crianças e a confiabilidade teste-reteste com 10 crianças com baixa visão. A consistência interna foi verificada a partir dos testes de todas as 40 crianças do estudo.

Foram adotados os seguintes critérios de inclusão: idade de dois até seis anos e seis meses; diagnóstico oftalmológico confirmado; procedimentos cirúrgicos e correção óptica já realizados; acuidade visual em ambos os olhos até 20/1000; desenvolvimento dentro dos padrões de normalidade, segundo triagem inicial da Fisioterapia e Terapia Ocupacional; exame neurológico normal. Todas as crianças foram avaliadas previamente por médicos oftalmologista e neuropediatra.

Quadro 1. Estudos sobre avaliação da visão funcional que reportaram análises de confiabilidade

\begin{tabular}{lcccc}
\hline Autor/ Data & Amostra & Confiabilidade & $\mathbf{n}$ & $\begin{array}{c}\text { Porcentagem } \\
\text { da amostra }\end{array}$ \\
\hline Blanksbye Langford & 193 & Interexaminadores & 30 & $15,5 \%$ \\
(1993) $^{(12)}$ & & Teste-reteste & 30 & $15,5 \%$ \\
Ricci et al. (2008)(10) $^{(50}$ & Interexaminadores & 20 & $40 \%$ \\
& & Teste-reteste & 10 & $20 \%$ \\
\hline
\end{tabular}

n= número de crianças que participaram da confiabilidade
Foram estabelecidos os seguintes critérios de exclusão: microcefalia, paralisia cerebral, atraso no desenvolvimento, mal-formação que dificultasse a motricidade, alteração de comportamento, déficit auditivo e lesão de vias ópticas.

A confiabilidade interexaminadores foi realizada entre as autoras do teste (Rossi LDF e Saliba GR), e entre a pesquisadora principal e uma acadêmica.

Para avaliar a confiabilidade teste-reteste, a AVIF-2 a 6 anos foi reaplicada com intervalo de 14 a 30 dias. A confiabilidade interexaminadores e a confiabilidade teste-reteste foram examinadas pela correlação de Pearson, para comparação com estudos prévios. Como o coeficiente de Pearson verifica apenas a correlação entre as variáveis, o que poderia gerar viés nos resultados, foi também utilizado o coeficiente de correlação intraclasse $(2,1)$, para avaliar a concordância teste-reteste e interexaminadores, com uso do MedCalc for Windows, versão 11.1.1.0 $0^{(15)}$

O coeficiente de correlação intraclasse foi adotado para verificar a concordância no escore total e nos domínios da AVIF-2 a 6 anos. Além disso, o coeficiente kappa ponderado foi calculado para cada um dos 47 itens do teste com uso do Statistical Package for Social Science - SPSS 12.0 (16).

A consistência interna dos itens foi examinada pelo coeficiente alfa de Cronbach (SPSS - 12.0), sendo também calculado o valor do coeficiente alfa por domínio, com exclusão de itens individuais. Os parâmetros para analisar alguns dos coeficientes utilizados neste estudo são(17):

- coeficiente de correlação intraclasse: excelente $(\geq 0,75)$; boa $(0,60$ a 0,74$)$; moderada $(0,40$ a 0,59); pobre $(<0,40)$;

- coeficiente kappa: excelente $(>0,80)$; boa $(0,61$ a 0,80); moderada $(0,41$ a 0,60$)$; baixa $(\leq 0,40)$;

- coeficiente alfa de Cronbach: boa consistência interna (0,70 a 0,90); baixa consistência interna $(<0,70)$.

\section{RESULTADOS}

Os valores de confiabilidade expressos pelo coeficiente de correlação linear de Pearson são apresentados na tabela 1.

Na confiabilidade teste-reteste calculada pelos valores da confiabilidade (CCI) (Tabela 2), ocorreu excelente confiabilidade para o valor total da AVIF-2 a 6 anos e para os domínios coordenação olho-mão e visão de cores. Constatou-se boa confiabilidade para seguimento visual e campo visual de confrontação. Confiabilidade moderada foi encontrada na fixação visual e pobre confiabilidade teste-reteste foi obtida para o domínio deslocamento no ambiente. Na visão de contraste houve concordância absoluta em $90 \%$ das avaliações e, nesse caso, o programa estatístico não fornece o $\mathrm{CCl}$.

O CCl entre as autoras do teste (Tabela 2) foi excelente para o total da AVIF-2 a 6 anos e para os domínios fixação visual, seguimento visual, coordenação olho-mão, deslocamento no ambiente e visão de cores. Ocorreu pobre confiabilidade para campo visual de confrontação; contudo, a visão de contraste apresentou concordância absoluta em $100 \%$ das avaliações.

Os (CCl entre o pesquisador principal e a acadêmica (Tabela 2) foram excelentes para o total da AVIF-2 a 6 anos e para seguimento visual, coordenação olho-mão, visão de contraste no plano, deslocamento no ambiente e visão de cores. Houve boa confiabilidade para campo visual de confrontação e moderada para fixação visual.

Os valores do coeficiente kappa ponderado para confiabilidade teste-reteste e interexaminadores de cada item da AVIF-2 a 6 anos variaram de -0,087 a 1,00 (Tabela 3). Os domínios com maior quantidade de itens com baixa confiabilidade, segundo o kappa, foram o seguimento visual, principalmente na confiabilidade teste-reteste, e o campo visual de confrontação, tanto na confiabilidade teste-reteste quanto na interexaminadores. Houve também baixa confiabilidade teste-reteste para os itens "realiza empilhamento de cubos" e "esbarra em objetos" no deslocamento no ambiente. 
Os valores da consistência interna analisados pelo coeficiente alfa de Cronbach são apresentados na tabela 4. Também foi verificado o valor de alfa com a retirada de itens isolados de cada domínio. O domínio que apresentou maior variação no valor de alfa, com a retirada de itens, foi o de coordenação olho-mão, no qual o alfa diminuiu de 0,77 para 0,50 com a retirada do item "alcance da conta preta" a $20 \mathrm{~cm}$ e, de 0,77 para 0,45 com a retirada do item "alcance da conta preta" a $40 \mathrm{~cm}$. Ao contrário, o alfa aumentou de 0,77 para 0,91 com a retirada do item "realiza empilhamento de cubos".

\section{DISCUSSÃO}

Poucos estudos para avaliação da visão funcional incluíram análises estatísticas para verificação da qualidade dos itens propostos. Na maioria deles foram utilizados somente roteiros sem a padronização necessária para um teste. As propriedades psicométricas da AVIF-2 a 6 anos foram analisadas, sendo sua confiabilidade estudada por meio de métodos estatísticos. Diferente dos estudos precedentes ${ }^{(1-10)}$, na amostra estudada todas as crianças do estudo encontravam-se na faixa etária de dois a seis anos e não tinham alterações neurológicas clínicas.

Comparado com os outros instrumentos, o VAP-CAP apresentou na confiabilidade interexaminadores valor igual a 0,99 para o total do teste, e de 0,91 a 1,00 nos domínios ${ }^{(2)}$, porém foi examinada apenas a correlação entre os escores. A confiabilidade interexaminadores

Tabela 1. Valores do coeficiente de correlação de Pearson para o escore total e por domínio da AVIF-2 a 6 anos

\begin{tabular}{|c|c|c|c|}
\hline \multirow[b]{2}{*}{$\begin{array}{l}\text { Confiabilidade escore total } \\
\text { e por domínio }\end{array}$} & \multirow{2}{*}{$\begin{array}{c}\text { Teste-reteste } \\
\text { A1/A2 } \\
\text { R }\end{array}$} & \multicolumn{2}{|c|}{ Interexaminadores } \\
\hline & & $\begin{array}{c}\mathrm{A} 1 / \mathrm{B} \\
\mathbf{R}\end{array}$ & $\begin{array}{l}\mathrm{A} 1 / \mathrm{C} \\
\mathrm{R}\end{array}$ \\
\hline Total AVIF-2 a 6 anos & 0,98 & 0,92 & 0,95 \\
\hline Fixação visual & 0,95 & 0,90 & 0,95 \\
\hline Seguimento visual & 0,70 & 0,86 & 0,86 \\
\hline Campo visual de confrontação & 0,74 & $0,53^{*}$ & 0,72 \\
\hline Coordenação olho-mão & 0,83 & 1,00 & 0,98 \\
\hline Visão de contraste & 1,00 & 1,00 & 1,00 \\
\hline Deslocamento no ambiente & $0,35^{*}$ & 1,00 & 1,00 \\
\hline Visão de cores & 1,00 & 1,00 & 1,00 \\
\hline
\end{tabular}

$\mathrm{A} 1=$ teste da pesquisadora principal; $\mathrm{A} 2=$ reteste; $\mathrm{B}=$ coautora do teste; $\mathrm{C}=$ acadêmica; $r=$ correlação de Pearson:

* = valor sem significância estatística, $p>0,05$

Tabela 2. Valores do coeficiente de correlação intraclasse para o total, e domínios da AVIF-2 a 6 anos

\begin{tabular}{lcccc}
\hline \multirow{2}{*}{$\begin{array}{l}\text { Confiabilidade escore total } \\
\text { e por domínio }\end{array}$} & \multicolumn{2}{c}{ Teste-reteste } & & \multicolumn{2}{c}{ Interexaminadores } \\
\cline { 2 - 2 } \cline { 5 - 6 } Total AVIF-2 a 6 anos & $\mathbf{A 1 / A 2}$ & & $\mathbf{A 1 / B}$ & $\mathbf{A 1 / C}$ \\
Fixação visual & 0,9353 & & 0,9197 & 0,9402 \\
Seguimento visual & 0,6361 & & 0,9068 & 0,5361 \\
Campo visual de confrontação & 0,6617 & & 0,3901 & 0,7158 \\
Coordenação olho-mão & 0,7692 & & 1,0000 & 0,9699 \\
Visão de contraste & $* *$ & & $* *$ & 1,0000 \\
Deslocamento no ambiente & 0,3721 & & 1,0000 & 1,0000 \\
Visão de cores & 1,0000 & & 1,0000 & 1,0000 \\
\hline
\end{tabular}

$\mathrm{A} 1=$ teste da pesquisadora principal; $\mathrm{A} 2=$ reteste; $\mathrm{B}=$ coautora do teste; $\mathrm{C}=$ acadêmica; ** $=$ resultados com $90 \%$ a $100 \%$ de concordância absoluta para o total da AVIF-2 a 6 anos com o coeficiente de correlação de Pearson foi de 0,92, entre suas autoras, e igual a 0,95 entre a autora principal e a acadêmica. Entretanto, a confiabilidade interexaminadores da AVIF-2 a 6 anos atingiu valores mais baixos por domínio que no VAP-CAP, sendo que em um deles, o campo visual de confrontação, foi de 0,53 e 0,72 . Os outros domínios apresentaram valor do coeficiente de correlação de Pearson acima de 0,86, ou seja, confiabilidade muito boa.

No teste-reteste, o VAP-CAP apresentou valores de correlação entre 0,50 e 1,00 nos domínios, e no total do teste de 0,97. Os valores de confiabilidade no teste-reteste da AVIF-2 a 6 anos variaram de 0,35 a 1,00. Em seis domínios, exceto o deslocamento no ambiente, que apresentou coeficiente igual a 0,35, esse valor ficou acima de 0,70 . Contudo, o valor do coeficiente de correlação de Pearson para o escore total foi de 0,98 (excelente).

Por meio do coeficiente de correlação intraclasse para o total da AVIF-2 a 6 anos e para seus domínios, verificou-se que em quatro domínios (seguimento visual, coordenação olho-mão, visão de contraste e visão de cores) a confiabilidade interexaminadores e teste-reteste foi boa a excelente. Além disso, o total da AVIF-2 a 6 anos manteve confiabilidade excelente em todas as análises com o CCl tanto no teste-reteste $(0,9353)$, quanto na confiabilidade interexaminadores $(0,9197$ e 0,9402). Os domínios com valor de confiabilidade pobre ou moderada (abaixo de 0,60 ) pelo CCl, na confiabilidade interexaminadores ou teste-reteste, foram fixação visual (na confiabilidade teste-reteste e interexaminadores), campo visual de confrontação (na confiabilidade interexaminadores) e deslocamento no ambiente (na confiabilidade teste-reteste).

Na análise isolada de cada um dos 47 itens da AVIF-2 a 6 anos, 0 coeficiente kappa ponderado apresentou confiabilidade baixa em quatro dos sete itens do seguimento visual (na confiabilidade teste-reteste), o que não existiu na confiabilidade interexaminadores. Baixa confiabilidade ocorreu ainda em itens do campo visual (na confiabilidade teste-reteste e interexaminadores), bem como nos itens "realiza empilhamentos de cubos" e "esbarra em objetos" (para confiabilidade teste-reteste). Deve-se enfatizar que em nenhum dos estudos prévios realizou-se análise por item, o que aumentaria o rigor do estudo.

Em consonância com esses dados, outros autores ${ }^{(9)}$ apontaram maior dificuldade no treinamento dos examinadores para avaliar o seguimento visual denominado "fluidity pursuit". Isso sugere que é difícil pontuar o domínio "seguimento visual", o qual necessita ser monitorado em trabalhos futuros.

Os domínios da AVIF-2 a 6 anos com baixa confiabilidade deverão ser revisados para garantir melhor uniformidade da medida. Em relação à fixação visual, sugere-se treinamento mais rigoroso na contagem do tempo de fixação. No campo visual de confrontação serão excluídos os itens de avaliação do campo visual superior, inferior-direito e inferior-esquerdo, pois apresentaram valores negativos de confiabilidade. Problemas tais como: fixação excêntrica, estrabismos, variações na posição compensatória de cabeça e alterações nas versões podem interferir na análise do campo de confrontação e explicar os baixos resultados alcançados na confiabilidade desse domínio.

Quanto ao item esbarra em objetos, houve diferença em algumas respostas dos pais no reteste. Para uma criança, a primeira informação foi dada pela mãe, e a segunda, pelo pai, o que pode ter interferido. Além disso, algumas mães mudaram a resposta na segunda avaliação. Para melhorar a confiabilidade teste-reteste, no domínio deslocamento no ambiente, as instruções de aplicação devem ser melhor especificadas.

Considerando-se o item realiza empilhamento de cubos, a criança ao colocar o primeiro cubo livremente sobre a superfície poderia, no momento do reteste, apresentar melhor resultado devido ao aprendizado, o que pode ter interferido na confiabilidade, uma vez que das 10 crianças que participaram do teste-reteste, 6 pontuaram 
Tabela 3. Valores do coeficiente kappa ponderado para os itens da AVIF-2 a 6 anos na confiabilidade teste-reteste e interexaminadores

\begin{tabular}{|c|c|c|c|}
\hline \multirow{2}{*}{$\begin{array}{l}\text { Confiabilidade } \\
\text { Item }\end{array}$} & \multirow{2}{*}{$\begin{array}{c}\text { Teste-reteste } \\
\text { A1/A2 }\end{array}$} & \multicolumn{2}{|c|}{ Interexaminadores } \\
\hline & & A1/B & A1/C \\
\hline Fixação a um objeto iluminado, a 20 cm & * & * & * \\
\hline Fixação ao pompom preto-e-branco, a $20 \mathrm{~cm}$ & $* *$ & $* *$ & $* *$ \\
\hline Fixação a um objeto pequeno, a $20 \mathrm{~cm}$ & * & 0,625 & * \\
\hline Fixação à figura da face, a 40 cm & * & * & 1,000 \\
\hline Fixação ao pompom preto-e-branco, a 40 cm & $* *$ & $* *$ & ** \\
\hline Seguimento horizontal do pompom, a $20 \mathrm{~cm}$ & 0,302 & 0,492 & 0,512 \\
\hline Seguimento vertical do pompom, a $20 \mathrm{~cm}$ & 0,265 & 0,437 & 1,000 \\
\hline Seguimento horizontal do pompom, a $40 \mathrm{~cm}$ & 0,324 & 0,679 & 0,681 \\
\hline Seguimento vertical do pompom, a $40 \mathrm{~cm}$ & 0,429 & 0,705 & 0,706 \\
\hline Busca visual após a queda, a $40 \mathrm{~cm}$ & $-0,154$ & 0,579 & 0,615 \\
\hline Campo visual superior & 0,074 & 0,286 & 0,400 \\
\hline Campo visual inferior/direito & 0,211 & $-0,125$ & 1,000 \\
\hline Campo visual inferior/esquerdo & $-0,154$ & $-0,125$ & $-0,111$ \\
\hline Realiza alcance do pompom, a $20 \mathrm{~cm}$ & ** & ** & ** \\
\hline Realiza alcance do objeto pequeno, a $20 \mathrm{~cm}$ & 0,474 & 1,000 & 1,000 \\
\hline Realiza alcance do pompom, a $40 \mathrm{~cm}$ & $* *$ & ** & ** \\
\hline Realiza alcance do objeto pequeno, a $40 \mathrm{~cm}$ & 0,571 & 1,000 & 0,865 \\
\hline Realiza empilhamento de cubos & 0,318 & 1,000 & 0,821 \\
\hline Realiza exploração do objeto & $* *$ & ** & ** \\
\hline Esbarra em objetos & $-0,087$ & 1,000 & 1,000 \\
\hline
\end{tabular}

$\mathrm{A} 1=$ teste da pesquisadora principal; $\mathrm{A} 2=$ reteste; $\mathrm{B}=$ coautora do teste; $\mathrm{C}=$ acadêmica; ${ }^{*}=$ um ou mais examinadores com respostas constantes, mas observou-se $90 \%$ ou mais de concordância absoluta entre as avaliações; ${ }^{* *}=100 \%$ de concordância absoluta

Tabela 4. Valores do coeficiente alfa de Cronbach para a AVIF-2 a 6 nos e seus domínios

\begin{tabular}{ll}
\hline Itens avaliados & alfa \\
\hline Total da AVIF-2 a 6 anos & 0,884 \\
Fixação visual & 0,845 \\
Seguimento visual & 0,882 \\
Campo visual de confrontação & $\mathbf{0 , 6 4 8}$ \\
Coordenação olho-mão & 0,777 \\
Visão de contraste & 0,973 \\
Deslocamento no ambiente & $\mathbf{0 , 5 8 4}$ \\
Visão de cores & 0,910 \\
\hline
\end{tabular}

Valores em negrito correspondem aos itens com baixa consistência interna

3 (empilha mais que quatro cubos) no primeiro teste e 9 pontuaram 3 no reteste. Das 40 crianças desse estudo, 36 obtiveram escore 3 no item realiza empilhamento de cubos.
Os domínios com baixa consistência interna foram campo visual de confrontação e deslocamento no ambiente. Contudo, os autores ressaltam que o deslocamento no ambiente foi constituído por apenas dois itens, o que pode ter interferido na consistência, já que um menor número de itens implica em consistência mais baixa. Boa consistência interna ocorreu no valor total da AVIF-2 a 6 anos e nos domínios fixação visual, seguimento visual, coordenação olho-mão e visão de cores.

\section{CONCLUSÕES}

A AVIF-2 a 6 anos apresenta excelente confiabilidade no escore total para documentar a visão funcional de crianças com baixa visão na faixa etária entre 2 e 6 anos de idade. A confiabilidade por domínios é boa em quatro, dos sete domínios avaliados (seguimento visual, coordenação olho-mão, visão de contraste no plano e visão de cores). Os resultados da AVIF-2 a 6 anos, associados à avaliação do desenvolvimento global podem fornecer informações confiáveis para elaboração do plano terapêutico da criança com baixa visão. É preciso considerar que alguns itens do AVIF-2 a 6 anos ainda podem ser aprimorados. 


\section{AGRADECIMENTOS}

Agradecemos, pela valiosa colaboração, às Profas. Érika Maria Parlato-Oliveira, Maria Elisabete R. F. Gasparetto e Heloisa G. R. G. Gagliardo.

\section{REFERÊNCIAS}

1. Droste PJ, Archer SM, Helveston EM. Measurement of low vision in children and infants. Ophthalmology. 1991;98(10):1513-8.

2. Blanksby DC, Langford PE. VAP-CAP: A procedure to assess the visual functioning of young visually impaired children. J Vis Impair Blind. 1993;87(1):46-9.

3. Katsumi O, Chedid SG, Kronheim JK, Henry RK, Jones CM, Hirose T. Visual Ability Score - a new method to analyze ability in visually impaired children. Acta Ophthalmol Scand. 1998;76(1):50-5.

4. Atkinson J, Anker S, Rae S, Hughes C, Braddick O. A test battery of child development for examining functional vision (ABCDEFV). Strabismus 2002;10(4):245-69.

5. Gagliardo HG, Goncalves VM, Lima MC. [A method to evaluate visual ability in infants]. Arq Neuropsiquiatr. 2004;62(2A):300-6. Portuguese.

6. Felius J, Stager DR Sr., Berry PM, Fawcett SL, Stager DR Jr, Salomao SR, et al. Development of an instrument to assess vision-related quality of life in young children. Am J Ophthalmol. 2004;138(3):362-72

7. Lopes MC, Salomao SR, Berezovsky A, Tartarella MB. Avaliação da qualidade de vida relacionada à visão em crianças com catarata congênita bilateral. Arq Bras Oftalmol. 2009;72(4):467-80
8. Birch EE, Cheng CS, Felius J. Validity and reliability of the Children's Visual Function Questionnaire (CVFO). J AAPOS 2007:11(5):473-9.

9. Mercuri E, Baranello G, Romeo DM, Cesarini L, Ricci D. The development of vision. Early Hum Dev. 2007:83:795-800.

10. Ricci D, Cesarini L, Groppo M, De Carli A, Gallini F, Serrao F, et al. Early assessment of visual function in full term newborns. Early Hum Dev. 2008;84(2):107-13.

11. Rossi LD, Vasconcelos GC, Saliba GR, Magalhães LC Soares AM, Cordeiro SS, et al. Avaliação da visão funcional para crianças com baixa visão de dois a seis anos de idade - estudo comparativo. Arq Bras Oftalmol. 2011;74(4):262-6.

12. Benson J, Schell BA. Measurement theory: application to occupational and physical therapy. In: Deusen J, Brunt D. Assessment in occupational therapy and physical therapy. Philadelphia: W. B. Saunders; 1997. p.3-24.

13. Cárdenas SF. Coeficientes de confiabilidad de instrumentos escritos em el marco de la teoría clásica de los tests [Internet]. Havana, Cuba; 2009. [cited 2009 Oct 22]. Available from: http://www.bvs.sld.cu/revistas/ems/vol22 2 2 08/ems06208.htm

14. Silveira CR, Menuchi MR, Simões DS, Caetano, MJ, Gobbi, LT. Validade de construção em testes de equilíbrio: ordenação cronológica na apresentação das tarefas. Rev Bras Cineantropom Desempenho Hum. 2006:8(3):66-72.

15. Medcalc: software de demonstração. Versão 11.1.1.0. Belgium: Mariakerke, 2004 [cited 2009 Nov 20]. Available form: http://www.medcalc.be

16. Stata statistical software. Versão 10.0. StataCorp, 2007 [Internet]. [cited 2008 Oct 20]. Available from: http://www.stata.com

17. Portney LG, Watkins MP. Foundations of clinical research: applications to practice. 3 aㅡ ed. Uper Saddle River, NJ: Pearson Education; 2008.

\section{xix congresso Latino-Americano de Estrabismo}

XIX Congresso Latino-Americano de Estrabismo

VI Congresso Brasileiro de Estrabismo

e Oftalmologia Pediátrica

V Congresso da Sociedade Latino-Americana

de Oftalmologia Pediátrica

\section{7 a 20 de abril de 2013}

Rio de Janeiro - RJ

Informações:

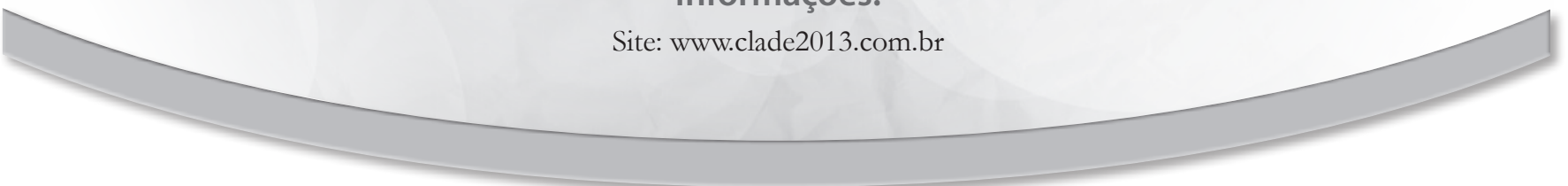

